STRESS MEDICINE

Stress Med. 15, 159-165 (1999)

\title{
LIPID LEVELS AND EMOTIONAL DISTRESS AMONG HEALTHY MALE COLLEGE STUDENTS
}

\author{
STEVEN M. SCHWARTZ, ${ }^{1 *}$ PhD, EDWARD P. SCHMITT, ${ }^{2}$ PsyD, MARK W. KETTERER, ${ }^{3}$ PhD \\ AND PETER C. TRASK, ${ }^{1} \mathrm{PhD}$ \\ ${ }^{1}$ Behavioral Medicine Program, University of Michigan, Ann Arbor, USA \\ ${ }^{2}$ Jenison Psychological Services, PC, USA \\ ${ }^{3}$ Henry Ford Health Sciences Center, Case Western Reserve, USA
}

\begin{abstract}
SUMMARY
Low lipid levels have been found in some studies to be associated with non-illness deaths (i.e. suicides, homicides and accidents). Likewise, low lipids have been associated with measures of emotional distress (e.g. anxiety, depression, hostility) in medical, psychiatric and forensic populations whose age, health status and/or personal habits make interpretation of the association problematic. The present study examined the relationship of lipid levels to emotional distress in young, healthy, male college students. To investigate possible confounding/mediating relationships, a number of clinical risk factors and demographic variables were also studied (age, drug use, alcohol use, nicotine use, exercise, obesity and resting hemodynamic values). Bivariate correlations showed that measures of emotional distress (SCL-90-R subscales, Toronto Alexithymia Scale) and clinical/demographic factors (alcohol use, age, blood pressure, weight and heart rate) were associated with lipid levels. In a hierarchical set multiple regression, only alcohol use, age, resting systolic blood pressure and the positive symptom total from the SCL-90-R were unique correlates of total cholesterol. These results add additional support to the growing evidence of an association between lipid levels and emotional functioning. Importantly, this relationship appears to exist apart from other risk factors. While various studies have focused on specific dimensions of emotional distress (i.e. anxiety, depression, hostility), the results of the present study suggest that more global measures of emotional distress might better account for the association with lipid levels. Copyright (C) 1999 John Wiley \& Sons, Ltd.
\end{abstract}

KEY WORDS - lipids; cholesterol; emotional distress; depression; hostility

The therapeutic effect of reduced cholesterol on the progression of cardiovascular disease has been well established. ${ }^{1,2}$ However, several observational studies and longitudinal, prospective treatment studies have found that overall mortality is not significantly reduced. ${ }^{3-6}$ Interestingly, some data suggest that reductions in fatal cardiovascular events are partially offset by several types of non-illness death (i.e. suicides, homicides and accidents). This controversial finding is further supported by a negative relationship between lipid levels and emotional distress variables (i.e. anxiety, depression, hostility). ${ }^{7-13}$ The negative association between lipids and emotional distress has been found in studies of forensic patients, ${ }^{14-17}$ psychiatric patients, ${ }^{7,10,11}$ heart diseased patients, ${ }^{9}$ and the general popula-

*Correspondence to: Dr S. M. Schwartz, Behavioral Medicine Program, University of Michigan Medical Center, 475 Market Place, Suite L, Ann Arbor, MI 48108, USA. tion. ${ }^{18-20}$ These data are also drawn from a broad range of geographic regions and diverse cultures. ${ }^{19-21}$

Importantly, the findings noted above are not particularly consistent, and a number of studies have either failed to find this relationship or found the opposite relationship (i.e. as emotional distress increased, so did lipid levels). ${ }^{22-28}$ For example, elevations of lipid levels have been reported in distressed individuals or during exposure to acute stressors. ${ }^{29-38}$ In some cases these results may reflect acute metabolic changes (e.g. decreased plasma volume) that accompany acute emotional arousal. $^{39}$ On the other hand, chronically low baseline cholesterol levels may have deleterious CNS effects, ${ }^{40}$ and have been found in animal studies to increase aggression. ${ }^{41}$ Three large treatment studies have failed to find increased nonillness deaths or significant emotional distress in their treatment arms. However, they also have 
methodological limitations relevant to this matter. For example, in some cases emotional status was not assessed in a standardized manner. ${ }^{26}$ Inclusion/ exclusion procedures in some studies (i.e. The West of Scotland study ${ }^{42}$; Weidner et al. ${ }^{28}$ ) confounded compliance with subject selection and intervention respectively. Since non-compliance is associated with psychiatric distress and substance abuse, both of these studies may have excluded relevant subgroups for addressing the non-illness death effect.

A number of questions have been raised regarding the relationship of lipid levels to emotional distress. ${ }^{43-45}$ For example, are specific lipid subfractions (i.e. LDL, HDL, triglycerides) responsible for this effect? ${ }^{19,21,46}$ Does the relationship hold up in an essentially young and healthy population? Recently Horsten et $a .^{8}$ studied the relationship between lipid levels and depressive symptoms in healthy middle-aged women and found that low serum cholesterol was associated with depressive symptomatology. Often emotional status is assessed in a limited fashion (i.e. narrow band assessment) and as such may not capture the range of negative emotional states that relate to lipid levels. Finally, temporal contiguity between the variable of interest (e.g. history of crime, suicide, accident, depression) and cholesterol assessment has often been lengthy. Therefore, studies attempting to link current lipid levels with 'history of' behaviors are subject to a number of intervening variables that limit causal value.

The present study sought to examine the relationship of several measures of naturally occurring lipid levels (i.e. total cholesterol, triglycerides, LDL, HDL) with current emotional status in a sample of young, healthy, male college students using both narrow and broad band assessment instruments of emotional distress and coping. Several of the measures used in this study were chosen as psychometrically sound clinical instruments that bear some theoretical and/or empirical relationship to cardiovascular disease (e.g. hostility, alexithymia). Clinical/demographic variables of potential relevance to cholesterol, and thus possible mediating/confounding variables, were also examined in order to better establish the unique relationship of emotional distress to cholesterol levels apart from other risk factors. It was hypothesized that some of the emotional distress assessment measures would show a statistically unique negative relationship to total serum cholesterol. The relationship to lipid subfractions was also investigated, but a priori hypotheses were not generated as this was considered exploratory.

\section{METHODOLOGY}

\section{Subjects}

Seventy-six male university students were initially recruited for participation. Eleven subjects (15 percent) did not complete the entire research protocol. One subject was dropped as an age outlier. Exclusion criteria included diabetes, hypertension, hypercholesterolemia or previous psychiatric history. One subject was dropped after admitting that he was being treated for hypercholesterolemia (i.e. lab values indicated total cholesterol of 297 and triglyceride level of 860), leaving a final sample of 63 subjects. The final sample represents a group of essentially healthy young males. The mean age was 20.03 years with a range from 18 to 24 years. Fifty-nine subjects identified themselves as Caucasian (93 percent), three as African American (5 percent) and one as Hispanic (2 percent). Relevant demographic and risk variables are presented in Table 1 and appear to indicate an active group of young adults with cholesterol levels falling well within accepted medical guidelines.

\section{Instrumentation}

The Cook Medley Hostility Scale ${ }^{47}$ is a 50 -item scale derived from the Minnesota Multiphasic Personality Inventory (MMPI), which has been shown to predict risk for development of coronary heart disease. ${ }^{48,49}$ The scale is thought to operationalize a number of constructs relevant to hostility and has demonstrated good convergent, discriminative and predictive validity.

The Symptom Checklist 90-Revised (SCL-90$\mathrm{R})^{50}$ is a multidimensional self-report instrument developed to assess a broad band of psychiatric symptomatology and is widely used in clinical practice and research. The checklist provides scores on nine psychological dimensions (i.e. somatization, obsessive-compulsive, interpersonal sensitivity, depression, anxiety, hostility, phobic anxiety, paranoid ideation, psychoticism) and three general indices (global severity index, positive symptom distress index and positive symptom total). This instrument was applied to assess gross psychiatric symptomatology and a variety of psychiatric manifestations of emotional distress. 
Table 1 - Means and standard deviations for clinical and demographic variables

\begin{tabular}{lrr}
\hline Variable & Mean & \multicolumn{1}{c}{ SD } \\
\hline Age (yr) & 20 & 1.8 \\
Body mass index & 25.7 & 4.2 \\
Resting heart rate (BPM) & 65.4 & 13.6 \\
RSBP & 120.1 & 10.1 \\
RDBP & 73.5 & 6.5 \\
Exercise (half hours per week) & 15.9 & 16.2 \\
Alcohol use (drinks per week) & 8.2 & 8.3 \\
Caffeine use (cups of coffee/tea, cans/ & 3.4 & 6.5 \\
$\quad$ glasses/bottles of pop per day) & & \\
Nicotine (cigarettes/cigars/chews/ & 0.8 & 1.7 \\
$\quad$ pipes full per day) & & \\
Total cholesterol & 166.1 & 35.3 \\
Triglycerides & 99.9 & 46.3 \\
LDL & 105.8 & 30.7 \\
HDL & 40.3 & 9.0 \\
\hline
\end{tabular}

RSBP, resting systolic blood pressure; RDBP, resting diastolic blood pressure; LDL, low density lipoprotein; HDL, high density lipoprotein.

Note: Lipid values are provided in $\mathrm{mg} / \mathrm{dl}$.

Alexithymia is a construct that reflects an individual's difficulty in recognizing and verbalizing emotional states. Alexithymia has a theoretical and empirical relationship to somatic illness. ${ }^{51}$ The Toronto Alexithymia Scale (TAS) ${ }^{52}$ was constructed as a psychometrically sound measure of the alexithymia construct based on a combination of empirical and theoretical rationales. The scale is composed of the following four factor analytically derived subscales: (1) difficulty identifying and distinguishing bodily sensations and feelings; (2) difficulty describing feelings; (3) reduced daydreaming; and (4) externally oriented thinking. The scale has shown 'adequate' reliability and validity. ${ }^{53-55}$

Blood serum assays were enzymatically analyzed with a Boehringer Mannheim/Hatachi 704 analyzer following the manufacturers' protocol for total serum cholesterol, triglycerides and high-density lipoproteins using absorbance measurements. Low-density lipoproteins were estimated using the Friedewald equation $(\mathrm{LDL}=$ total cholesterol triglycerides $/ 5+\mathrm{HDL}$ ). Lipid subfractions were derived in order to explore the possibility that one or more of these indices may bear a relationship to measures of emotional distress.

\section{Procedures}

Phase I. Following initial recruitment and informed consent, subjects completed the battery of self-report measures including the Cook-Medley Hostility Scale, SCL-90-R and Toronto Alexithymia Scale. The first phase of the study was completed after subjects were given oral and written instruction for completing Phase II.

Phase II. Subjects reported to the university health services laboratory within 48 hours of Phase I to have height and weight measured, blood pressure taken and to provide a blood sample (the consent form included an outline of the potential risks and benefits of a venipuncture procedure). Venipuncture was performed using a \#21 needle following standard venipuncture protocol by a certified medical technologist employed at the health services center. Blood draws were conducted between the hours of $8.00 \mathrm{am}$ and 12 noon following a $12-14$-hour fast. Ten milliliters of venous blood were obtained for testing and analyzed by the phlebotomy lab at a local community hospital.

\section{RESULTS}

The means and standard deviations for the psychometric dependent measures are provided in Table 2. Bivariate tests of association (Pearson product-moment correlation coefficients) were run between the lipid values (i.e. total cholesterol, triglycerides, LDL, HDL) with the demographic and risk variables, and the emotional distress measures. There were no meaningfully significant correlations of any variable with the lipid subfractions. The clinical/demographic factors significantly $(p<0.05$ or less) associated with total cholesterol included alcohol use (0.47), age (0.44), weight (0.29), resting systolic blood pressure $(0.27)$ and resting heart rate $(0.27)$. These variables are commonly accepted risk factors for cardiovascular disease. Among the psychometric measures, several indices from the SCL-90-R (positive symptom total, -0.30 and paranoid ideation, -0.26$)$, and scale 2 of the Toronto Alexithymia Scale $(-0.29)$ were negatively associated with total cholesterol. The positive symptom total summary scale reflects the frequency of distressing symptoms, and paranoia concerns a projection of hostility and suspiciousness about the motives and intentions of others. Scale 2 from the TAS concerns the inability to label affective states in oneself. Importantly, these bivariate associations were not corrected for 
Table 2 - Means and standard deviations for psychometric variables

\begin{tabular}{lcc}
\hline Variable & Mean & SD \\
\hline SCL-90-R & & \\
$\quad$ Somatization & 0.51 & 0.53 \\
Obsessive-compulsive & 0.94 & 0.74 \\
Interpersonal sensitivity & 0.85 & 0.72 \\
Depression & 0.86 & 0.73 \\
Anxiety & 0.54 & 0.54 \\
Hostility & 0.72 & 0.78 \\
Phobic anxiety & 0.25 & 0.39 \\
Psychoticism & 0.48 & 0.57 \\
GSI & 0.68 & 0.52 \\
PSDI & 1.57 & 0.48 \\
PST & 0.35 & 0.21 \\
Toronto Alexithymia Scale & 58.86 & 10.87 \\
TAS 1 & 18.00 & 4.76 \\
TAS 2 & 19.03 & 5.78 \\
TAS 3 & 11.40 & 3.35 \\
TAS 4 & 13.59 & 3.56 \\
Cook-Medley Hostility Scale & 22.89 & 7.87 \\
\hline
\end{tabular}

Note: GSI, Global severity index; PSDI, positive symptom distress index; PST, positive symptom total; TAS, Toronto Alexithymia Scale.

repeated significance testing and are therefore subject to Type I error inflation.

In order to determine the unique relationship of the variables with significant bivariate correlations to total cholesterol and control for Type I error, a hierarchical set regression using total cholesterol as the criterion was employed. The solution for the regression equation is shown in Table 3 . The first set was defined using the significant demographic/ risk factors (i.e. age, alcohol use, weight, resting systolic blood pressure, resting heart rate) in order to statistically control for these variables. The second set included the emotional distress measures with significant bivariate associations (i.e. paranoia and positive symptom total subscales of the
SCL-90-R, and Scale 2 of the TAS). Variables within the two sets were entered in a stepwise fashion. While it is acknowledged that stepwise entry capitalizes on chance, it was decided that clinical pragmatism and the exploratory nature of our a priori questions dictated finding the best predictive solution. In addition, the hierarchical set approach is a more conservative strategy and partially corrects for the more liberal aspect of stepwise entry. An $R^{2}$ adjusted for shrinkage is also provided in Table 3. Predictor variables with the strongest residual relationship (largest beta) were entered if the $p$ value was less than or equal to 0.05 . Previously entered predictor variables were removed if the adjusted $p$ value was greater than 0.10 . Interestingly, the hierarchical set regression equation made use of age, alcohol use, resting systolic blood pressure (RSBP) and SCL-90-R positive symptom total as the only significant predictors of total cholesterol. The final model including alcohol use, age, RSBP and positive symptom total accounted for 48 percent of the variance in this sample's total cholesterol scores $\left(R^{2}=0.484\right)$. The beta weights were positive for the first three predictors, indicating that greater alcohol use, older age and higher RSBP are associated with increased total cholesterol. However, positive symptom total displayed a negative beta weight, indicating that increasing frequency of psychological symptoms is strongly and independently associated with lower total serum cholesterol. Importantly, while positive symptom total only accounted for approximately 7 percent of the variance, this is after controlling for the other associated variables.

\section{DISCUSSION}

These data lend additional support to the notion that there is a relationship between total cholesterol

Table 3 - Multiple regression predicting total cholesterol from clinical/demographic and psychometric variables which yielded significance in the bivariate tests

\begin{tabular}{lccccc}
\hline Variable & $B$ & Beta & $R^{2}$ change & $F$ change & Significance \\
\hline Alcohol & 0.31 & 0.45 & 0.22 & 17.02 & 0.001 \\
Age & 6.55 & 0.33 & 0.15 & 14.18 & 0.001 \\
RSBP & 0.56 & 0.17 & 0.05 & 5.05 & 0.03 \\
PST & -0.42 & -0.27 & 0.07 & 7.53 & 0.008 \\
\hline
\end{tabular}

RSBP, Resting systolic blood pressure; PST, positive symptom total.

Note: Multiple $R=0.70 ; R^{2}=0.49$; adjusted $R^{2}=0.45$. 
level and some measures of emotional distress. Importantly, this study replicates the negative relationship between cholesterol and emotional distress in a young, active and essentially healthy male sample without demonstrable cardiovascular disease or related medical risk factors (e.g. essential hypertension). The relationship did not, however, hold up for any of the lipid subfractions (LDL, HDL, triglycerides). The strong relationships between alcohol use, age and RSBP and total cholesterol are consistent with previous findings and represent commonly accepted clinical correlates of cardiac care. ${ }^{56}$ This finding also lends credence to the validity of the sample and the methodology. It is somewhat surprising to find age such a powerful predictor of total cholesterol given that the age variable in this sample was so truncated. Perhaps late adolescence/early adulthood is a period of change in cholesterol metabolism? It is somewhat interesting to note the unique contribution of a more global index of distress. The positive symptom total index reflects the frequency of self reported psychological distress symptoms. Given this was the only measure of emotional distress to be retained in the hierarchical regression equation (along with age), it may be that more general measures of emotional distress better account for the heterogeneity of negative psychological sequelae associated with low lipid levels. This may also partially account for the mixed findings in the literature such that the precise emotional manifestation of low lipid levels is mediated by as yet unidentified mediating variables. Therefore, studies employing only a narrow band assessment of emotional status may miss identification of this relationship.

The correlational nature of these data is a limiting factor. This is true for most of the studies cited, but when taken together with published clinical trials examining non-illness death, ${ }^{44}$ they provide a provocative stimulus but no firm conclusions. Further, the relevance of gender was not addressed here and remains an essentially unexplored aspect of this controversial literature. Additional research is indicated in order to obtain a more thorough understanding of this association and establish more causal relationships. Future studies might monitor healthy normals and clinical cases prospectively for changes in both mood and behavior during the use of antilipidemic medications, or during clinically or experimentally imposed diet changes. The use of standard reversal designs (i.e. ABA) is ideal for this type of test.

\section{REFERENCES}

1. Grundy, S. M. Cholesterol and coronary heart disease: Future directions. J. Am. Med. Assoc. (1990); 264: 3053-3059.

2. Manninen, V., Elo, O., Frick, H. et al. Lipid alterations and decline in the incidence of coronary heart disease. Circulation 1988; 86: 1-11.

3. Frick, M. H., Elo, M. O., Happa, K., Heinonen, O. P., Heinsalmi, P. et al. Helsinki heart study: Primary prevention trail with gomfibrozil in middle aged men with dyslipidemia: Safety of treatment, changes in risk factors, and incidence of coronary heart disease. New Engl. J. Med. 1987; 317: 1237-1245.

4. Neaton, J. D., Blackburn, H., Jacobs, D. et al. Serum cholesterol level and mortality findings for men screened in the Multiple Risk Factor Intervention Trial. Arch. Intern. Med. 1992; 152: 14901500.

5. Lipid Research Clinics Program. The Lipid Research Clinics coronary primary prevention trial results I. Reduction in incidence of coronary heart disease. J. Am. Med. Assoc. 1984; 251: 351-364.

6. Zureik, M., Courbon, D., Ducimetiere, P. Serum cholesterol concentration and death from suicide in men: Paris prospective study I. Brit. Med. J. 1996; 313: 649-651.

7. Golier, J. A., Marzuk, P. M., Leon, A. C., Weiner, C. and Tardiff, K. Low serum cholesterol and attempted suicide. Am. J. Psych. 1995; 152: 419-423.

8. Horsten, M., Wamala, S.P., Vingerhoets, A. and Orth-Gomer, K. Depressive symptoms, social support, and lipid profile in healthy middle-aged women. Psychosom. Med. 1997; 59: 521-528.

9. Ketterer, M. W., Brymer, J., Rhoads, K., Kraft, P., Goldberg, A. D. and Lovallo W. R. Lipid-lowering therapy and violent death: Is depression a culprit? Stress Med. 1994; 10: 233-237.

10. Modai, I., Valevski, A., Dror, S. and Weizman, A. Serum cholesterol levels and suicidal tendencies in psychiatric inpatients. J. Clin. Psychiat. 1994; 55(6): 252-254.

11. Morgan, R. E., Palinkas, L. A., Barrett-Connor, E. L. and Wingard, D. L. Plasma cholesterol and depressive symptoms in older men. Lancet 1993; 341: 75-79.

12. Santiago, J. M. and Dalen, J. E. Cholesterol and violent behavior. Arch. Intern. Med. 1994; 154: 1317-1321.

13. Sullivan, P. F., Joyce, P. R., Bulik, C. M., Mulder, R. T. and Oakley-Brown, M. Total cholesterol and suicidality in depression. Biol. Psychiat. 1994; 36: 472-477.

14. Hillbrand, M. and Foster, H. G. Serum cholesterol and severity of aggression. Psychol. Rep. 1993; 72: 270. 
15. Hillbrand, M., Spitz, R. T. and Foster, H. G. Serum cholesterol and aggression in hospitalized male forensic patients. J. Behav. Med. 1995; 18: 33-43.

16. Virkkunen, M. Serum cholesterol in antisocial personality. Neuropsychobiology 1979; 5: 27-30.

17. Virkkunen, M. Serum cholesterol levels in homicidal offenders. Biol. Psychiat. 1979; 10: 65-69.

18. Irvine, M. J. and Logan, A. G. Is knowing your cholesterol number harmful? J. Clin. Epidemiol. 1994; 47(2): 131-145.

19. Lindberg, G., Larsson, G., Setterlind, S. and Rastam, L. Serum lipids and mood in working men and women in Sweden. J. Epidemiol. Commun. Health 1994; 48: 360-363.

20. Lindberg, G., Rastam, L., Gullberg, B. et al. Low serum cholesterol concentrations and short term mortality from injuries in men and women. Brit. Med. J. 1992; 305: 277-279.

21. Jacobs, D., Blackburn, H., Higgins, M. et al. Report of the conference on low blood cholesterol and mortality associations. Circulation 1992; 86: 10461060 .

22. Brown, S. L., Salive, M. E., Harris, T. B., Simonsick, E. M. Guralnik, J. M. and Kohout, F. J. Low cholesterol concentrations and severe depressive symptoms in elderly people. Brit. Med. J. 1994; 308: $1328-1332$.

23. Freedman, D. S., Byers, T., Barrett, D. H., Stroup, N. E., Eaker, E. and Monroe-Blum, H. Plasma lipid levels and psychologic characteristics in men. Am. J. Epidemiol. 1995; 141: 507-517.

24. Gould, A. L., Rossouw, J., Santanello, N. C., Heyse, J. F. and Furberg, C. D. Cholesterol reduction yields clinical benefit: A new look at old data. Circulation 1995; 91: 2274-2282.

25. Iribarren, C., Reed, D. M., Wergowske, G., Burchfield, C. M. and Dwyer, J. H. Serum cholesterol level and mortality due to suicide and trauma in the Honolulu Heart Program. Arch. Intern. Med. 1995; 155: 695-700.

26. Scandinavian Simvastatin, Survival Study Group randomized trial of cholesterol lowering in 4444 patients with coronary heart disease: The Scandinavian Simvastatin Survival Study (4S). Lancet 1994; 344: 1383-1389.

27. Vartiainen, E., Puska, P., Pekkanen, J., Tuomilehto, J., Lonnqvist, J. and Ehnholm, C. Serum cholesterol concentration and mortality from accidents, suicide, and other violent causes. Brit. Med. J. 1994; 309: 445-447.

28. Weidner, G., Conner, S. L., Hollis, J. F. and Conner, W. E. Improvements in hostility and depression in relation to dietary change and cholesterol lowering. Ann. Intern. Med. 1992; 117: 820-823.

29. Dimsdale, J. E. and Herd, J. A. Variability of plasma lipids in response to emotional arousal. Psychosom. Med. 1982; 44: 413-430.
30. Dujovne, V. F. and Houston, B. K. Hostility-related and plasma lipids. J. Behav. Med. 1991; 14: 555-565.

31. Greene, R. E., Houston, B. K. and Holleran, S. A. Aggressiveness, dominance, developmental factors and serum cholesterol level in college males. $J$. Behav. Med. 1995; 18: 569-578.

32. Johnson, E. H., Collier, P., Nazzaro, P. and Gilbert, D. C. Psychological and physiological predictors of lipids in black males. J. Behav. Med. 1992; 15: 285-289.

33. Lundberg, U., Hedman, M., Melin, B. and Frankenhaeuser, M. Type A behavior in healthy males and females as related to physiological reactivity and blood lipids. Psychosom. Med. 1989; 51: 113-122.

34. McCann, B. S., Warnick, G. R. and Knopp, R. H. Changes in plasma and dietary intake accompanying shifts in perceived workload and stress. Psychosom. Med. 1990; 52: 97-108.

35. Niaura, R., Herbert, P. N., McMahon, N. and Sommerville, L. Repressive coping and blood lipids in men and women. Psychosom. Med. 1992; 54: 698-706.

36. Weidner, G., Hutt, J., Connor, S. L. and Mendell, N. R. Family stress and coronary risk in children. Psychosom. Med. 1992; 54: 471-479.

37. Weidner, G., Sexton, G., McLellern, R., Connor, S. L. and Matarazzo, J. D. The role of Type A behavior and hostility in an elevation of plasma lipids in adult women and men. Psychosom. Med. 1987; 49: 136-145.

38. Wiist, W. H. and Flack, J. M. A test of the John Henryism hypothesis: cholesterol and blood pressure. J. Behav. Med. 1992; 15: 15-22.

39. Patterson, S. M., Gottdiener, J. S., Hecht, G., Vargot, S. and Krantz, D. S. Effects of acute mental stress on serum lipids: mediating effects of plasma volume. Psychosom. Med. 1993; 55: 525-532.

40. Benton, D. Do low cholesterol levels slow mental processing? Psychosom. Med. 1995; 57: 50-53.

41. Kaplan, J. R., Manuck, S. B. and Shively, C. The effects of fat and cholesterol on social behavior in monkeys. Psychosom. Med. 1991; 53: 634-642.

42. Shepherd, J., Cobbe, S. M., Ford, I. et al. for the West of Scotland Coronary Prevention Study Group. Prevention of coronary heart disease with pravastatin in men with hypercholesterolemia. New Engl. J. Med. 1995; 333: 1301-1307.

43. Hawthon, K., Cowen, P., Owens, D., Bond, A. and Elliot, M. Low serum cholesterol and suicide. Brit. $J$. Psychiat. 1993; 162: 818-825.

44. Muldoon, M. F., Roussouw, J. E., Manuck, S. B., Glueck, C. J., Kaplan, J. R. and Kaufmann, P. G. Low or lowered cholesterol and risk of death from suicide and trauma. Metabolism 1993; 42: 45-56.

45. Schwartz, S. M. and Ketterer, M. W. Cholesterol lowering and emotional distress: Current status and future directions. In: Lipids, Health and Behavior. Hillbrand, M. and Spitz, R. (Eds) American 
Psychological Association, Washington, DC, 1997, pp. 113-122.

46. Stensvold, I., Urdal, P., Thurmer, H., Tverdal, A., Lund-Larsen, P. G. and Foss, O. P. High-density lipoprotein cholesterol and coronary, cardiovascular and all cause mortality among middle-aged Norwegian men and women. Eur. Heart J. 1992; 13: $1155-1163$.

47. Cook, W. W. and Medley, D. M. Proposed hostility and pharisaic-virtue scales for the MMPI. J. Appl. Psychol. 1954; 38: 414-418.

48. Barefoot, J. C., Peterson, B. L., Dahlstrom, W. G., Siegler, I. C., Anderson, N. B. and Williams, R. B. Hostility patterns and health implications: Correlates of Cook-Medley Hostility Scale scores in a national survey. Health Psychol. 1991; 10: 18-24.

49. Williams, R. B. and Barefoot, J. C. Coronary-prone behavior: The emerging role of the hostility complex. In: Type A Behavior Pattern: Research, Theory, and Intervention. Houston, B. K and Snyder, C. R. (Eds) Wiley, New York, 1988, pp. 189-211.

50. Derogatis. SCL-90R Manual II. Clinical Psychometric Research, Baltimore, MD, 1983.
51. Taylor, G. J., Bagby, R. M. and Parker, J. D. A. The alexithymia construct: A potential paradigm for psychosomatic medicine. Psychosomatics 1991; 32: $153-164$.

52. Taylor, G. J., Ryan, D. P. and Bagby, R. M. Toward the development of a new self-report alexithymia scale. Psychother. Psychosom. 1985; 44: 191-199.

53. Bagby, R. M., Taylor, G. J. and Ryan, D. Toronto Alexithymia Scale: Relationship with personality and psychopathology measures. Psychother. Psychosom. 1986; 45: 207-215.

54. Bagby, R. M., Taylor, G. J. and Parker, J. D. A. Construct validity of the Toronto Alexithymia Scale. Psychother. Psychosom. 1988; 50: 29-34.

55. Bagby, R. M., Taylor, G. J., Parker, J. D. A. and Loiselle, C. Cross-validation of the factor structure of the Toronto Alexithymia Scale. J. Psychosom. Res. 1990; 34: 47-51.

56. Farmer, J. A. and Gotto, A. M. Risk factors for coronary artery disease. In: Heart Disease: A Textbook of Cardiovascular Medicine. Braunwald, E. (Ed.) W. B. Saunders, Philadelphia, PA, 1993. 